\title{
An RFID-Based Access and Location Service for Pervasive Grids
}

\author{
Antonio Coronato $^{2}$, Gennaro Della Vecchia ${ }^{1}$, and Giuseppe De Pietro ${ }^{1}$ \\ ${ }^{1}$ ICAR-CNR, Via Castellino 111, 80131 Napoli, Italy \\ \{giuseppe.depietro, gennaro.dellavecchia\}@na.icar.cnr.it \\ ${ }^{2}$ SASIT-CNR, Via Castellino 111, 80131 Napoli, Italy \\ coronato.a@na.drr.cnr.it
}

\begin{abstract}
Grid computing and pervasive computing have rapidly emerged and affirmed as paradigms for high performance computing and for user-friendly computing respectively. These two separate worlds, however, can now interact each other in such a way that both pervasive and grid computing communities can benefit from integrating the two paradigms into a whole. This conjunction is already taking place to yield the pervasive grid computing model, and the present paper is focused on this approach. Purposely, it describes an Access\&Location service for pervasive grid applications which uses RFID and WiFi technologies to grant access and locate mobile objects within a pervasive environment. Since this service is compliant to the OGSA specifications, it can be easily integrated into classic grid environments, therefore enhancing them with pervasive capabilities.
\end{abstract}

\section{Introduction}

During the last decade, new computing models have emerged and rapidly affirmed. In particular, terms like Grid Computing and Pervasive Computing have become of common use not only in the scientific and academic world, but also in business fields.

The Grid computing model proved to be an effective way to face very complex problems. The term "The Grid" was primarily introduced by Foster and Kesselman to indicate a distributed computing infrastructure for advanced science and engineering [1]. Successively, it has been extended to denote the virtualization of distributed computing and data resources such as processing, network bandwidth and storage capacity to create a single system image, granting users and applications seamless access to vast IT capabilities. As a result, Grids are geographically distributed environments, equipped with shared heterogeneous services and resources accessible to users and applications for solving complex computational problems and accessing big storage spaces.

On the other hand, the goal of Pervasive Computing lies in the development of environments where highly heterogeneous hardware and software components can seamlessly and spontaneously interoperate, in order to provide a variety of services to users independently of the specific characteristics of the environment and of the client devices [2]. Therefore, mobile devices should come into the environment in a natural way, as their owner moves through it, and interactions should occur in a transparent 
and proactive manner - that is, the owner will not have to carry out explicit operations for being able to approach services and resources, and the environment has to be able to self-adapt and self-configure in order to host and handle incoming mobile devices.

These two worlds are now evolving towards a common paradigm, namely the Pervasive Grid Computing [8] where the final target is to build grid environments that have characteristics typically found in pervasive systems. As a matter of fact, from the Grid Computing community's point of view, it is now time to integrate mobile devices into the grid because mobile devices are increasingly used for accessing services in any distributed environment. Moreover, it is possible to enhance the QoS of existing Grid services with characteristics like the context-awareness and the proactivity, which are proper of the Pervasive Computing. From the other side's perspective, Pervasive Computing environments can proficiently benefit from grid technologies both to interconnect existing and emerging pervasive environments and to build and deploy new services that require high performance computing and large data resources [7].

This paper is organized as follows. Section 2 discusses some motivations and related works. Section 3 describes the architecture of the Access\&Location service. Section 4 describes a running scenario. Finally, section 6 concludes the paper.

\section{Motivations and Related Works}

\subsection{Motivations}

For a long while, mobile and wireless devices have not been seen as useful resources by traditional Grid environments. However, if we recall the Metcalfe's law, which claims that the usefulness of a network-based system proportionally grows with the number of active nodes, and also consider that mobile devices capabilities have been substantially improved over the time, it can be rightly stated that mobile and wireless devices are now of interest for the Grid community [3].

In particular, they may and can be incorporated into the Grid either as service/ resource consumers or as service/resource providers [5].

Obviously, such an integration is not costless [6]. This is mainly due to the fact that current Grid middleware infrastructures do not support mobile devices, first because they do not take into account any pervasive requirements like heterogeneity, spontaneity, transparency, context-awareness etc., and then because i) they are still too much heavy to be implemented on mobile and wearable equipments, ii) they are not network-centric, i.e. they assume fixed TCP/IP connections and do not deal with wireless networks and other mobile technologies and iii) they typically support only one interaction paradigm, i.e. SOAP messaging, whereas the Pervasive model requires a variety of mechanisms [9].

\subsection{Other Related Works}

Over the last years, some valuable efforts have been done in making Grid architecttures able to support wireless technologies and mobile devices. In particular, the paradigm of Mobile Grid or Wireless Grid has been proposed [3-6]. More recently, this paradigm has evolved into the Pervasive Grid model [7-8], which still aims at 
making Grid environments able to integrate mobile devices, but within a pervasive framework, which is to say in seamless and transparent ways to the user. Moreover, the final objective is to enhance Grid environments with characteristics the like of context-awareness and pro-activity that are typically found in Pervasive environments.

This effort has been officially formalized in the year 2003, when Ubicomp-RG -a Global Grid Forum Research Group- was established in order to explore the feasibility of synergy between Pervasive and Grid communities.

Some interesting works towards the realization of Pervasive Grids have been done and are here reported.

In [4] mobile devices are viewed as active resources for the Grid. In particular, the authors developed a software infrastructure for deploying Grid services on mobile nodes and making them active actors in the Grid. This solution relies on a lightweight version of the .NET framework, namely the .NET Compact Framework, which enables to deploy on mobile devices simple Grid Services that require limited amount of resources. It is important to note that such a solution applies only to mobile devices equipped with the Microsoft Pocket PC operating system and requires several manual operations for installation and configuration.

In [9] authors argued that the SOAP messaging, which is the current interaction paradigm for standard grid middleware infrastructures, is not well suited for pervasive grids. They developed several plug-ins plus a handling component for enlarging the set of available interaction mechanisms in order to make grids able to support heterogeneous software components.

Another middleware infrastructure for pervasive grids has been presented in [10]. In that case, authors have concentrated their effort on the extension of existing resource manager components in grid applications for making them able to register mobile devices.

In recent years, the fast growing RFID technology, initially developed for applications in logistics and industry, suggested to integrate mechanisms for locating and tracking mobile objects based on RFID tagged entities in a pervasive computing environment. Many papers can be found in literature dealing with this novel approach.

In [11], a location sensing prototype system that uses Radio Frequency Identification (RFID) technology for locating objects inside buildings is presented, where the authors demonstrate that active RFID is a viable and cost-effective candidate for indoor location sensing.

In [12] the authors analyze the general interaction patterns in pervasive computing settings using Bluetooth-Enabled Active Tags and Passive RFID Technology together with Mobile Phones, and show how information about the user's context is derived by a combination of active and passive tags present in the user's environment.

In [13], based on the pervasive deployment of RFID tags, the authors propose a novel ubiquitous architecture followed by a protocol for tracking mobile objects in real-time, and give delay analysis and simulation results.

In [14] and [15] the author presents a general-purpose infrastructure for building and managing location-aware applications in ubiquitous computing settings, with the goal of providing people, places, and objects with computational functionalities to support and annotate them. 


\subsection{Our Contribution}

Our contribution consists of a set of basic functionalities that provide a grid environment with some pervasive characteristics. In particular, the realized service supports the following requirements:

a. Locating RFID mobile objects - RFID tagged objects are located within the environment and integrated as active resources in the grid;

b. Locating WiFi enabled mobile devices - WiFi mobile devices are located within the environment;

c. Detecting leaving RFID and WiFi mobile objects - Leaving RFID tagged objects and WiFi mobile devices are detected in order to reliably handle pending computations and allocated resources.

\section{Access and Location Service}

This service is able to locate active mobile objects like WiFi enabled devices and RFID tagged entities. It offers both locating and location functions, that is, the function Locate_Object returns the position of a specific object, whereas the function Get_Objects returns the list of objects that are active at a specific location. Moreover, the service uses an asynchronous communication mechanism for notifying the environment of the following events:

1. NEW_DEVICE - This event is notified when a new WiFi enabled device enters the environment;

2. NEW_LOCATION - This event is notified when a WiFi enabled device moves in a new location of the environment;

3. DEVICE_HAS_LEFT - This event is notified when a WiFi enabled device leaves the environment;

4. RFID_OBJECT - This event is notified when an RFID tagged object is detected by an RFID antenna. The event carries an information on the physical location of the object.

5. RFID_OBJECT_HAS_LEFT - This event is notified when a previously detected RFID tagged object is no more sensed by the RF antenna.

Such events are logically grouped in a class named LOCALIZATION.

In addition to location and locating functions, Access\&Location service provides basic network connectivity facilities for incoming mobile devices and detects leaving objects.

This service's architecture consists of two layers with the following components:

- WiFiLocatingComponent - This component is in charge of locating WiFi enabled mobile devices. In particular, a WiFi location is identified by the area covered by a specific wireless Access Point (AP). Within the environment, one WiFiLocatingComponent is deployed for each wireless AP. Current implementation uses 3Com Office Connect Wireless 11g Access Points. Whenever a mobile device connects with the AP, the AP writes an 


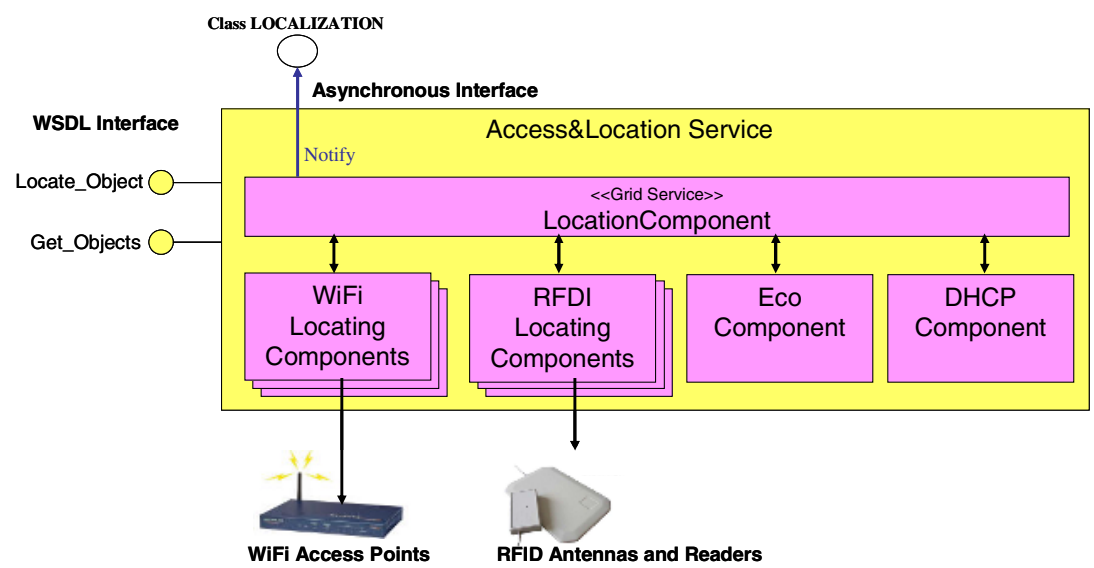

Fig. 1. Interfaces and architecture of the Access\&Location Service

event on its log file. The WiFiLocatingComponent periodically interrogates such a log file and updates the LocationComponent when a new device has been detected. The WiFiLocatingComponent maintains information on devices locally connected to its AP.

- RFIDLocatingComponent - This component is in charge of locating RFID tagged objects. An RFID location is identified by the area covered by the RF antenna of a specific RFID reader. Current implementation uses the passive, short-range, Feig Electronic model ISC.MR 100/101. When a tagged object is sensed by the antenna, the RFID reader generates an event that is caught by the RFIDLocatingComponent which, in turn, updates the LocationComponent.

- DHCPComponent - This component implements a DHCP service. It provides network connectivity to incoming IP enabled devices as a standard DHCP, but it has also additional functionalities. In particular, it is able to release an IP address on demand. In this way, if a device has left the environment, the LocationComponent requires that the DHCPComponent free the IP address of that device.

- EcoComponent - This component sends ping messages towards mobile IP devices in order to detect leaving objects. When an implicit disconnection is detected, the component communicates such an event to the LocationComponent that notifies the DEVICE_HAS_LEFT event.

- LocationComponent - This component is in charge of handling global location states obtained by combining information coming from the underlying components. In particular, it notifies the environment with events of the class LOCALIZATION.

In addition to these components, we are developing BluetoothLocatingComponents in order to grant access and locate Bluetooth enabled devices. 


\section{Experimental Scenario}

The experimental scenario consists of a physical site located in a three-floors building. The virtual environment uses two floors of the building.

Floor zero has a computing laboratory in which a cluster of 24 linux PCs, a 12 processors Silicon Graphics workstation and a motion capture system are deployed. Such resources are collected in a wired grid built at the top of the Globus Toolkit 4.0 platform.

On floor two, wireless access to the grid is available. Here, two 3Com Office Connect Wireless $11 \mathrm{~g}$ Access Points identify two distinct locations. L1 is a student laboratory where our students develop their activities and periodically perform ETests. L2 is a multimedia room equipped with a projector, an interactive monitor, and other multimedia devices.

Some services are available:

- MotionCaptureService - This service relies on the motion capture system. An actor (equipped with optical markers) moves around in the multimedia laboratory. Several cameras capture his movements that are reproduced on a graphic station. The graphic station shows a skeleton, which moves accordingly with the actor, and records data movement in a file;

- RenderingService - This service enables users to submit row motion data and to build 3D graphic applications. This service is exposed as a Grid Service and is available at every location (L1, L2);

- PresentationService - This service enables a user to project its presentation in the multimedia room. The service receives a pdf/ppt file via a dialog form and then enables speaker to control the presentation flow. This is an interactive service, which requires the speaker to be in the room for presentation. As a consequence, the service must be available only in the multimedia room (L2);

- ETestingService - This service performs on-line evaluation tests for courseware activities. When a session test starts, students must be in the student laboratory. Evaluation tests are synchronized and students have a predefined length of time for completing each test section. Students can interrupt their test by explicitly closing the service or by leaving the multimedia room. This service is exposed as a Grid Service, but it must be available only in the student laboratory (L1).

In this environment, services availability depends on user location, rights, and context. Purposely, we can report some example scenarios:

1. The PresentationService is available only to users that are located in L2. In particular, a WiFi mobile device, who moves in location L2, is located by the location service that notifies the environment. From now on, the mobile user can access the PresentationService.

2. The ETestingService is available to every mobile user in L1, who holds a personal RFID tag. In particular, while a test session is active, a mobile user that enters L1 can get access to the service. At the entrance, the mobile user is recognized by an RFID reader, then he is invited to sit to a specific desk that has already been configured for the e-test with the information of the incoming 

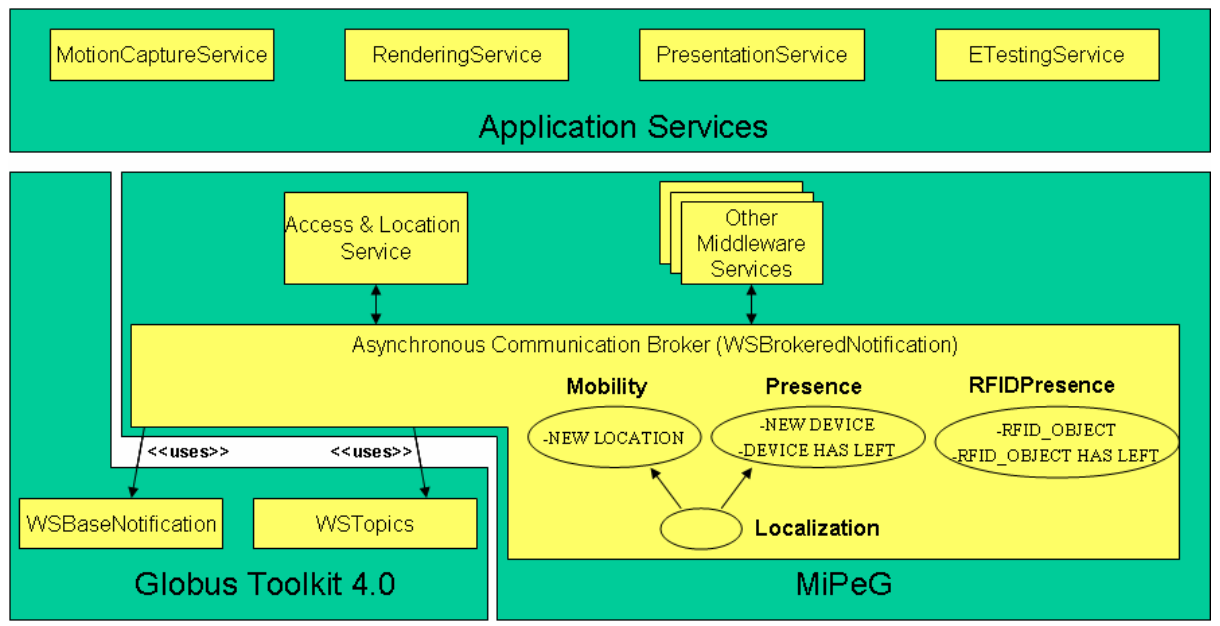

Fig. 2. General architecture of a pervasive grid computing environment

user. On the other hand, if the user leaves its desk, this is detected by the RFID reader and notified to the environment. Then, the ETestingService automatically disconnects the leaving user and denies any further tentative of reconnection.

3. The RenderingService is available to authorized users at every location. The QoS of this service is enhanced with context-awareness features. Indeed, when a mobile user launches such a service while in location L1, rendered data are reproduced on his mobile device. Henceforth, if the user moves in the multimedia room (L2), rendered data are automatically switched on the interactive monitor (if idle).

In the previous example, we have shown how our Grid environment already can draw benefits from location-awareness.

In addition, we are realizing an advanced multimedia service for immersive virtual worlds by integrating into a whole 1) the MotionCaptureService, 2) the Real-Time RenderingService, 3) RFID locating technologies, and other wearable equipments like multimedia helmets and gloves. In particular, the service consists of a virtual world projected to a mobile user by means of an helmet. The mobile user interacts with the virtual world, which is rendered in real time by a Grid service. User actions and movements are caught and notified to the rendering service, which consequently builds a new scene. This is just another example of new application service that can be realized by combining Pervasive and Grid Computing.

\section{Conclusions}

This paper is focused on pervasive grid environments, a novel approach aimed to combine together both the grid and the pervasive computing models, and is intended to show the benefits that the conjunction of these two worlds can yield.

Purposely, we have presented an Access\&Location service that enhances a real grid environment by augmenting its features with pervasive capabilities. 
Such a service relies on WiFi and RFID technologies. In particular, it i) grants access to WiFi devices; ii) locates WiFi and RFID mobile objects; iii) detects leaving WiFi and RFID objects; iv) enables WiFi and RFID mobile objects to be registered as active objects for the grid and, more generally, v) enhances the QoS of existing services with location-awareness and allows for the realizations of new services.

\section{References}

[1] I. Foster, C. Kesselman, "The Grid: Blueprint for a New Computing Infrastructure". Morgan Kaufmann, 1999.

[2] D. Saha and A. Murkrjee, "Pervasive Computing: A Paradigm for the 21st Century", IEEE Computer, March 2003.

[3] L. W. McKnight, J. Howinson, S. Bradner, "Wireless Grids", IEEE Internet Computing, July-August 2004.

[4] D. C. Chu and M. Humphrey, "Mobile OGSI.NET: Grid Computing on Mobile Devices", International Workshop on Grid Computing, GRID 2004.

[5] B. Clarke and M. Humphrey, "Beyond the 'Device as Portal': Meeting the Requirements of Wireless and Mobile Devices in the Legion of Grid Computing System", International Parallel and Distributed Processing Symposium, IPDPS 2002.

[6] T. Phan, L. Huang and C. Dulan, "Challenge: Integrating Mobile Devices Into the Computational Grid", International Conference on Mobile Computing and Networking, MobiCom 2002.

[7] N. Daves, A. Friday, and O. Storz, "Exploring the Grid's Potential for Ubiquitous Computing", IEEE Pervasive Computing, April-June 2004.

[8] V. Hingne, A. Joshi, T. Finin, H. Kargupta, E. Houstis, "Towards a Pervasive Grid", International Parallel and Distributed Processing Symposium, IPDPS 2003.

[9] G. Coulson, P. Grace and G. Blair, D. Duce, C. Cooper and M. Sagar, "A Middleware Approach for Pervasive Grid Environments", Workshop on Ubiquitous Computing and eResearchNational eScience Centre, Edinburgh, UK 18-19 May 2005.

[10] C. F. R. Geyer et. al., "GRAPEp: Towards Pervasive Grid Executions", III Workshop on Computational Grids and Applications, WCGA 2005.

[11] Lionel M. Ni1, Yunhao Liu, Yiu Cho Lau and Abhishek P. Patil, "LANDMARC: Indoor Location Sensing Using Active RFID", Proceedings of the First IEEE International Conference on Pervasive Computing and Communications (PerCom'03), 0-7695-1895/03 (C) 2003 IEEE

[12] Frank Siegemund and Christian Florkemeier, "Interaction in Pervasive Computing Settings using Bluetooth-Enabled Active Tags and Passive RFID Technology together with Mobile Phones", Proceedings of the First IEEE International Conference on Pervasive Computing and Communications (PerCom'03), 0-7695-1893-1/03 @ 2003 IEEE

[13] Pradip De, Kalyan Basu and Sajal K. Das, "An Ubiquitous Architectural Framework and Protocol for Object Tracking using RFID Tags", Proceedings of the First Annual International Conference on Mobile and Ubiquitous Systems: Networking and Services (MobiQuitous'04), 0-7695-2208-4/04 \$20.00 @ 2004 IEEE

[14] Ichiro Satoh, "Linking Physical Worlds to LogicalWorlds with Mobile Agents", Proceedings of the 2004 IEEE International Conference on Mobile Data Management (MDM'04) 0-7695-2070-7/04 @ 2004 IEEE

[15] Ichiro Satoh, “A Location Model for Pervasive Computing Environments”, Proceedings of the 3rd IEEE Int'l Conf. on Pervasive Computing and Communications (PerCom 2005), 0-7695-2299-8/05 @ 2005 IEEE 